\title{
Erratum to: Immersion autometallographic demonstration of pathological zinc accumulation in human acute neural diseases
}

Lin Zhu • Yong Tang • Han-dong Wang •

Zhi-yuan Zhang $\cdot$ Hao Pan

Published online: 17 March 2013

(C) Springer-Verlag Italia 2013

Erratum to: Neurol Sci (2012) 33(4):855-861

DOI 10.1007/s10072-011-0847-2

The publisher regrets that Fig. 1d was printed incorrectly in the published issue due to the negligence of author.

The correct figure is displayed below:

The online version of the original article can be found under doi:10.1007/s10072-011-0847-2.

L. Zhu · Y. Tang $\cdot$ H. Wang $(\bowtie) \cdot$ Z. Zhang $\cdot$ H. Pan Department of Neurosurgery, School of Medicine, Jinling Hospital, Nanjing University, 305 East Zhongshan Road, 210002 Nanjing, Jiangsu Province, People's Republic of China e-mail: wanghandong61@126.com 

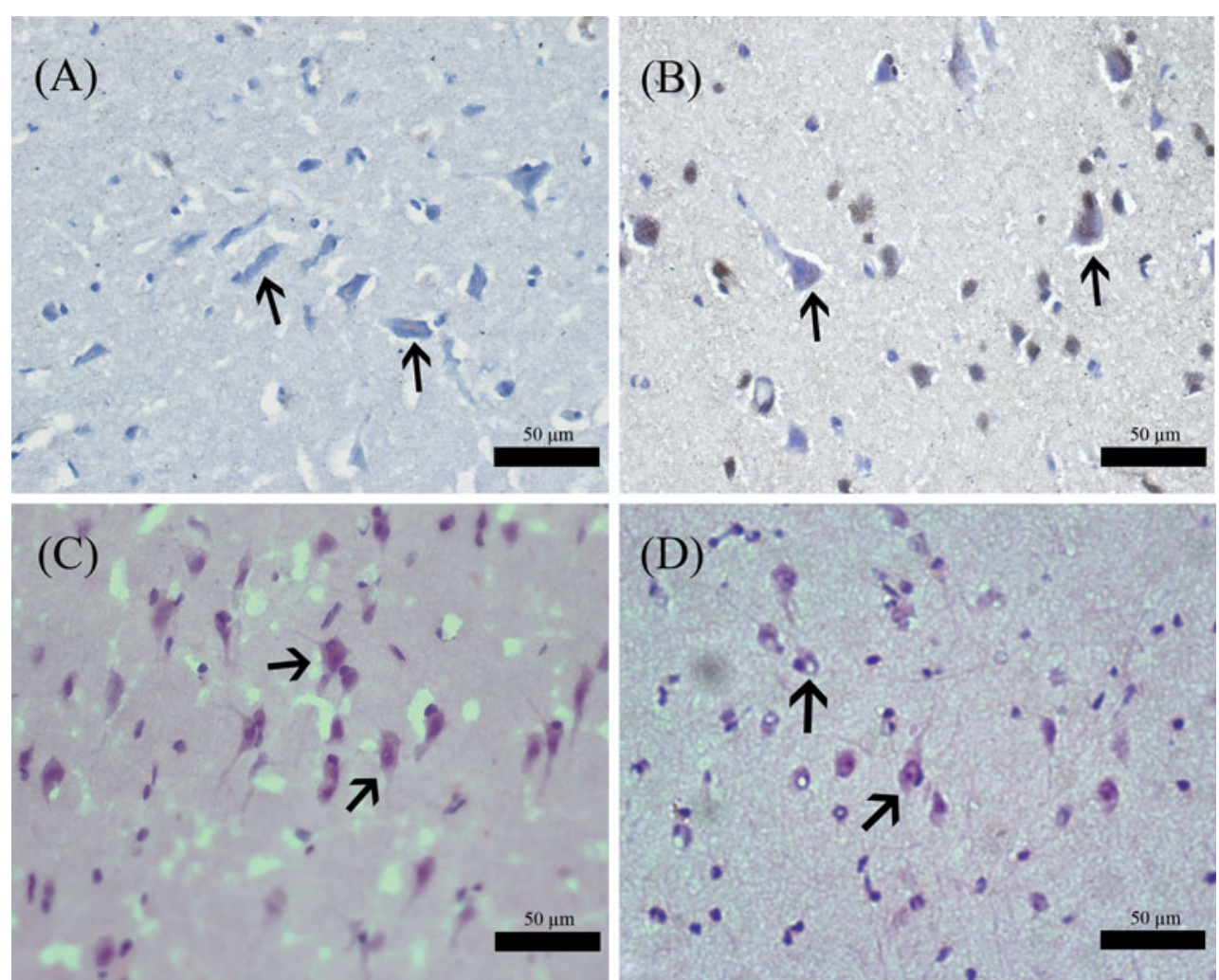

Fig. 1 Zinc accumulation and neuronal degeneration in human temporal cortical neurons following acute carotid artery infarction. Human cortex sections from normal brain $(\mathbf{a}, \mathbf{c})$ or from ischemia temporal $(\mathbf{b}, \mathbf{d})$ tissue were stained with AMG $(\mathbf{a}, \mathbf{b})$ and H\&E (c, d). Compare with the normal cortex (a), large amounts of AMG

enhanced zinc-sulphur nanocrystals positive neurons were seen in the ischemia brains (b). Sections of the same samples were stained with $H \& E$. Under $H \& E$, these AMG-positive neurons show injured appearance, e.g. vacuolar degeneration and karyolysis. All sections were $15 \mu \mathrm{m}$ thick. Scale bar $50 \mu \mathrm{m}$ 